\title{
Taxifolin protects rat against myocardial ischemia/reperfusion injury by modulating the mitochondrial apoptosis pathway
}

Zhenqiu Tang ${ }^{1}$, Chunjuan Yang ${ }^{2,3}$, Baoyan Zuo ${ }^{4}$, Yanan Zhang ${ }^{1}$, Gaosong Wu ${ }^{1}$, Yudi Wang ${ }^{1}$, Zhibin Wang Corresp. 1

1 Key Laboratory of Chinese Materia Medica, Heilongjiang University of Traditional Chinese Medicine, Harbin, Heilongjiang, China

2 College of Pharmacy, Harbin Medical University, Harbin, Heilongjiang, China

3 Beijing Shunyue Technology Co., Ltd., Beijing, China

4 School of Pharmacy, Shenyang Pharmaceutical University, Shenyang, Liaoning, China

Corresponding Author: Zhibin Wang

Email address: wzbmailbox@hljucm.net

Background: Taxifolin (TAX), is an active flavonoid, that plays an underlying protective role on the cardiovascular system. This study aimed to evaluate its effect and potential mechanisms on myocardial ischemia/reperfusion (I/R) injury. Methods: Healthy rat heart was subjected to I/R using the Langendorff apparatus. Hemodynamic parameters, including heart rate (HR), left ventricular developed pressure (LVDP), maximum/minimum rate of the left ventricular pressure rise $\left(+d p / d t_{\max }\right.$ and $\left.-d p / d t_{\min }\right)$ and rate pressure product (RPP) were recorded during the perfusion. Histopathological examination of left ventricular was measured by hematoxylin-eosin (HE) staining. Creatine kinase-MB (CK-MB) and lactate dehydrogenase (LDH) activities in the effluent perfusion, and the levels of malondialdehyde (MDA), superoxide dismutase (SOD) and glutathione peroxidase (GSH$\mathrm{PX}$ ) in the tissue were assayed. Apoptosis related proteins, such as B-cell lymphoma-2 (Bcl2 ), Bcl2-associated X (Bax) and cytochrome $\mathrm{c}(\mathrm{Cyt}-\mathrm{c})$ were also assayed by ELISA. Western blot was employed to determine apoptosis-executive proteins, including caspase 3 and 9. TUNEL assay was performed to evaluate the effect TAX on myocardial apoptosis. Results: TAX significantly improved the ventricular functional recovery, as evident by the increase in LVDP, $+d \mathrm{dp} / \mathrm{dt}_{\max },-d \mathrm{dp} / \mathrm{dt}_{\min }$ and RPP, The levels of SOD, GSH-PX were also increased, but those of LDH, CK-MB, and MDA were decreased. Furthermore, TAX up-regulated the Bcl-2 protein level but down-regulated the levels of Bax, Cyt-c, caspase 3 and 9 protein, thereby inhibits the myocardial apoptosis. Discussion: TAX treatment remarkably improved the cardiac function, regulated oxidative stress and attenuated apoptosis. Hence, TAX has a cardioprotective effect against I/R injury by modulating mitochondrial apoptosis pathway. 


\section{Taxifolin protects rat against myocardial \\ 2 ischemia/reperfusion injury by modulating the \\ 3 mitochondrial apoptosis pathway}

4

5 Zhenqiu Tang ${ }^{1, \dagger}$, Chunjuan Yang ${ }^{2,3, \dagger}$, Baoyan Zuo ${ }^{4}$, Yanan Zhang ${ }^{1}$, Gaosong Wu ${ }^{1}$, Yudi Wang ${ }^{1}$, 6 Zhibin Wang ${ }^{1}$,*

$8{ }^{1}$ Key Laboratory of Chinese Materia Medica, Heilongjiang University of Chinese Medicine,

9 Harbin, Heilongjiang, China

${ }^{2}$ College of Pharmacy, Harbin Medical University, Harbin, Heilongjiang, China

${ }^{3}$ Beijing Shunyue Technology Co., Ltd., Beijing, China

${ }^{4}$ School of Pharmacy, Shenyang Pharmaceutical University, Shenyang, Liaoning, China

† These authors contribute equally to this paper.

Corresponding Author:

Zhibin Wang

No. 24 Heping road, Harbin, Heilongjiang, 150040, China

Email address: wzbmailbox@hljucm.net

\section{ABSTRACT}

Background: Taxifolin (TAX), is an active flavonoid, that plays an underlying protective role on the cardiovascular system. This study aimed to evaluate its effect and potential mechanisms on myocardial ischemia/reperfusion (I/R) injury.

Methods: Healthy rat heart was subjected to $\mathrm{I} / \mathrm{R}$ using the Langendorff apparatus. Hemodynamic parameters, including heart rate (HR), left ventricular developed pressure (LVDP), maximum/minimum rate of the left ventricular pressure rise $\left(+d p / d t_{\max }\right.$ and $\left.-\mathrm{dp} / \mathrm{dt}_{\min }\right)$ and rate pressure product (RPP) were recorded during the perfusion. Histopathological examination of left ventricular was measured by hematoxylin-eosin (HE) staining. Creatine 
29 kinase-MB (CK-MB) and lactate dehydrogenase (LDH) activities in the effluent perfusion, and

30 the levels of malondialdehyde (MDA), superoxide dismutase (SOD) and glutathione peroxidase

31 (GSH-PX) in the tissue were assayed. Apoptosis related proteins, such as B-cell lymphoma-2

32 (Bcl-2), Bcl2-associated $\mathrm{X}(\mathrm{Bax})$ and cytochrome $\mathrm{c}$ (Cyt-c) were also assayed by ELISA.

33 Western blot was employed to determine apoptosis-executive proteins, including caspase 3 and

34 9. TUNEL assay was performed to evaluate the effect TAX on myocardial apoptosis.

35

36

37

38

39

40

41

42

43

44

45

46

47

48

49

50

51

52

53

54

55

56

57

58

59

60

61

62

63

64

65

Results: TAX significantly improved the ventricular functional recovery, as evident by the increase in LVDP, $+d p / d t_{\max }$, $-d p / d t_{\min }$ and RPP, The levels of SOD, GSH-PX were also increased, but those of LDH, CK-MB, and MDA were decreased. Furthermore, TAX upregulated the Bcl-2 protein level but down-regulated the levels of Bax, Cyt-c, caspase 3 and 9 protein, thereby inhibits the myocardial apoptosis.

Discussion: TAX treatment remarkably improved the cardiac function, regulated oxidative stress and attenuated apoptosis. Hence, TAX has a cardioprotective effect against I/R injury by modulating mitochondrial apoptosis pathway.

3

\section{INTRODUCTION}

Ischemic heart disease is a threat to human health. Extracorporeal circulation and coronary bypass surgery are usually employed to improve myocardial ischaemia after myocardial infarction occurs. The timely restoration of blood flow to the ischemic myocardium (reperfusion) became the standard treatment for these patients. However, reperfusion may cause additional heart damage. This condition is referred to as cardiac ischaemia/reperfusion (I/R) injury (Braunwald et al., 2012). The reperfusion of ischaemic tissues is often associated with microvascular dysfunction. The mechanisms may involve the release of oxygen radicals and inflammatory mediators (Carden et al., 2000). Nowadays, tissue injury induced by I/R is a major factor, which often cause death. During myocardial I/R injury, cardiomyocytes undergo death at an increased frequency, mainly including necrosis and apoptosis (Gottlieb et al., 1994). Apoptosis involves programmed cell death, which is the vital pathological process in acute reperfusion injury (Konstantinidis et al., 2012). When the amount of cardiomyocyte decreases, the heart may undergo ventricular remodelling, compensatory cardiac hypertrophy and eventually lead to heart failure (Pangonyte et al., 2008; Du et al., 2010). Therefore, exploring the detailed mechanisms that trigger cardiomyocyte death and the means to prevent it during $I / R$ injury is still a public issue.

In $\mathrm{I} / \mathrm{R}$ injury, morphological changes in myocardial tissue can be observed, including microvascular damage and myocardial cell edema. The symptoms of $\mathrm{I} / \mathrm{R}$ include myocardial enzyme release, arrhythmias and weak systolic function (Naito et al., 2000). Generally, this reperfusion damage is caused by increased free radical activity. When circulating blood decreases, the level of oxygen supply cannot sustain the oxygen demand by cardiomyocytes, and 
66

67

68

69

70

71

72

73

74

75

76

77

78

79

80

81

82

83

84

85

86

87

88

89

90

91

92

93

94

95

96

97

98

99

100

101

102

103

the aerobic metabolism turns into anaerobic metabolism (Giordano et al., 2005). Anaerobic metabolism leads to the production of lactic acid which results in disturbances in ionic homeostasis. A timely reperfusion is crucial for the recovery of an ischemic myocardium, but by the sudden re-appearance of circulating blood to the dying myocardium, oxygen species (ROS) will be produced as a response to hyperoxia which can worsen the functional situation of the organization (Akhlaghi et al., 2009).

Flavonoid is the most prevalent class of naturally occurring compound and is ubiquitous in woody and herb plants. It exerts multiple biochemical properties and wide pharmacological effects (Moon et al., 2006). Epidemiological studies have shown that flavonoid is associated with a reduced risk of cardiovascular diseases (Raj Narayana et al., 2001; Bjorklund et al., 2017). Fisetin, a plant-derived bioflavonoid, significantly attenuated I/R-induced tissue injury, blunted the oxidative stress, and restored mitochondrial structure and function (Shanmugam et al., 2018). Quercetin has been demonstrated to improve post ischemic recovery of the isolated heart of rats after global ischemia (Barteková et al., 2010). TAX exerts anti-inflammatory effects and prevents oxidative stress-induced injury in human endothelial cells (Guo et al., 2015) and rat peritoneal macrophages (Arutyunyan et al., 2016). It also possesses free radical scavenging, antioxidant and anti-inflammatory effects (Sun et al., 2014; Xie et al., 2017). TAX is structurally similar to quercetin. Hence, we suspect that it also has a beneficial effect on the cardiovascular system. Recent studies demonstrated that TAX could inhibit cardiac hypertrophy and attenuate ventricular fibrosis after pressure overload. These beneficial effects were at least mediated by suppressing oxidative stress and the excess production of ROS. (Guo et al., 2015; Sun et al., 2014). However, the potential of TAX for $I / R$ protection remains unclear. TAX is a potential candidate for the prevention or treatment for $\mathrm{I} / \mathrm{R}$ injury. However, the influence of TAX on the injury of $I / R$ in isolated rat hearts has not been reported. In this study, we aimed to evaluate the cardioprotective effects of TAX and investigated the mechanisms underlying these effects in isolated hearts of rats.

Myocardial ischemic events are unpredictable. The clinical application of pre-conditioning drugs is limited. Therefore, researchers turned to a new endogenous protective strategy, which is post-conditioning. In 2006, the protective effect of post-conditioning in ischemic reperfusion on cerebral ischemia was first reported (Zhao et al., 2006). Subsequent studies have validated the protective effect of post-conditioning in various global ischemia models ex vivo and ischemia and hypoxia models in vitro. Flavonoids reduce the injury of myocardial ischemia on perfusion in a post-conditioning way (Wang et al., 2017; Xuan et al., 2016). Therefore, we adopted the post-conditioning method in this study.

\section{MATERIALS \& METHODS}

\section{Experimental animals and treatment}
Male SD rats (280-300 g each) were obtained from the Laboratory Animal Center of 
104 Heilongjiang Medicine University Medical (License Number: SCXK (hei) 2013-004). The rats

105

106

107

108

109

110

111

112

113

114

115

116

117

118

119

120

121

122

123

124

125

126

127

128

129

130

131

132

133

134

135

136

137

138

139

140

141

142

143

were housed under standard conditions with natural light $(12 \mathrm{hr})$ and dark $(12 \mathrm{hr})$ at $22 \pm 2{ }^{\circ} \mathrm{C}$. Rats were fed with common laboratory chow and allowed to drink tap water ad libitum during the experimental period. The investigation conformed to Guide for the Care and Use of Laboratory Animals (revised, 1996, http://dels.nas.edu/resources/static-assets/ilar/miscellaneous /GUIDE1996.pdf). All animal experiments were approved by the College of Pharmacy of Heilongjiang University of Chinese Medicine, Animal Ethics Committee (Approval number: SYXK (hei)-2013-012).

\section{Reagents and antibodies}

TAX (purity $\geq 98 \%$ ) was purchased from Sigma-Aldrich (St. Louis, MO, USA). Creatine kinase-MB (CK-MB), lactate dehydrogenase (LDH), malondialdehyde (MDA) glutathione peroxidase (GSH-PX) and superoxide dismutase (SOD) assay kit were obtained from Nanjing Jiancheng Bioengineering Institute (Jiangsu, China). The enzyme-linked immunnosorbent assay kit about mitochondrial apoptosis [B-cell lymphoma-2 (Bcl-2), Bcl2-associated X (Bax) and cytochrome c (Cyt-c)] was obtained from Cloud-Clone Corp (Houston, TX, USA). Monoclonal primary antibodies anti- $\beta$-actin, anti-active caspase 3 and 9 were purchased from Abcam (MA, USA). Fluorescent-labeled goat anti-rabbit IgG secondary antibody was obtained from LI-COR Biosciences (Lincoln, Nebraska, USA).

\section{Experimental protocol}

TAX was dissolved in ethanol $(15.21,45.63 \mathrm{mg} / \mathrm{ml})$ and then transferred into Krebs-Henseleit (K-H) solution. Ethanol solution $(0.1 \mathrm{~mL})$ was added into $1 \mathrm{~L} \mathrm{~K}-\mathrm{H}$ solution. The final concentration was 5 or $15 \mu \mathrm{M}$. Rats were randomly divided into four groups $(\mathrm{n}=8)$ : Normal control group (Control); Myocardial I/R control group (I/R); I/R + TAX $5 \mu \mathrm{M}$ treatment group (TAX $5 \mu \mathrm{M}$ ); I/R + TAX $15 \mu \mathrm{M}$ treatment group (TAX $15 \mu \mathrm{M}$ ). The experimental protocol is shown in Fig. 1. Control group: The hearts were subjected a continuous perfusion of $\mathrm{K}-\mathrm{H}$ solution for $120 \mathrm{~min}$. I/R group: The hearts were perfused for $30 \mathrm{~min}$ to stabilization. Subsequently, global ischemia was performed at $37{ }^{\circ} \mathrm{C}$ for $30 \mathrm{~min}$, followed by reperfusion with $\mathrm{K}-\mathrm{H}$ solution for $60 \mathrm{~min}$. TAX $5 \mu \mathrm{M}$ group: The hearts were perfused for $30 \mathrm{~min}$ to stabilization. Subsequently, global ischemia was performed for $30 \mathrm{~min}$ at $37{ }^{\circ} \mathrm{C}$, followed by reperfusion with $5 \mu \mathrm{M}$ of TAX-saturated $\mathrm{K}-\mathrm{H}$ solution for $60 \mathrm{~min}$. TAX $15 \mu \mathrm{M}$ group: The hearts were perfused for $30 \mathrm{~min}$ to stabilization. Subsequently, global ischemia was performed for $30 \mathrm{~min}$, followed by reperfusion perfused with $15 \mu \mathrm{M}$ of TAX-saturated $\mathrm{K}-\mathrm{H}$ solution for $60 \mathrm{~min}$.

\section{Langendorff preparation}

After anesthetization via intraperitoneal injection (chloral hydrate solution, $300 \mathrm{mg} / \mathrm{kg}$ ), rat hearts were quickly removed and subsequently perfused in the Langendorff apparatus. The perfusion was performed for $30 \mathrm{~min}$ in a modified $\mathrm{K}-\mathrm{H}$ buffer gassed with $95 \% \mathrm{O}_{2}$ and $5 \% \mathrm{CO}_{2}$ at a constant flow velocity and constant temperature $\left(37^{\circ} \mathrm{C}\right)$. The composition of $\mathrm{K}-\mathrm{H}$ buffer as 
144 the following (mM): $\mathrm{NaCl} 118, \mathrm{MgSO}_{4} 1.2, \mathrm{KCl} 3.2, \mathrm{NaHCO}_{3} 25, \mathrm{KH}_{2} \mathrm{PO}_{4} 1.18, \mathrm{CaCl}_{2} 2.5$ and 145 glucose 5.5. After equilibration, $30 \mathrm{~min}$ global ischemia was induced followed by $60 \mathrm{~min}$ of 146 reperfusion. Control group utilized the same protocol, but no ischemic induction was used. 147 Water-filled balloon that is inserted into the left ventricular cavity was used to monitor 148 hemodynamic parameters. The left ventricular end-diastolic pressure (LVEDP) was maintained 149 at 5-10 $\mathrm{mmHg}$ by adjusting the size and position of the balloon. The whole procedure was 150 completed within $2 \mathrm{~min}$. The inclusion criteria of experimental samples were a heart rate (HR) of $151>250$ beats/min and a left ventricular developed pressure (LVDP) of $>75 \mathrm{mmHg}$ in equilibrium 152 period. In the experiment, we prepared 12 rats in each group with an average of three or four 153 failures. Finally, only eight rats from each group were used in the subsequent experiment. The 154

155

156

157

158

159

160

161

162

163

164

165

166

167

168

169

170

171

172

173

174

175

176

177

178

179

180

181

182

183 hemodynamic parameters were recorded during perfusion, including heart rate (HR), LVDP, the maximum/minimum rate of left ventricular pressure rise $\left(+d p / d t_{\max }\right.$ and $\left.-d p / d t_{\min }\right)$, which are important indices to evaluate the left ventricular systolic and diastolic function. Rate pressure product $(\mathrm{RPP})=\mathrm{HR} \times$ LVDP. After 60 minutes of reperfusion, heart tissue was taken from Langendorff apparatus. The left ventricles of three hearts were cut from each group and then fixed in $10 \%$ neutral formalin. The other tissues were immediately placed in the freezer at -80 ${ }^{\circ} \mathrm{C}$.

\section{Histopathological evaluation of left ventricle sections}

For histopathological examination, the cut left ventricle of heart tissues were fixed in $10 \%$ neutral formalin at room temperature. After 2 hours, the tissue piece was embedded in paraffin. Next, the piece was cut into $3 \mu \mathrm{m}$ thick tissue sections, after which it was subjected to hematoxylin-eosin (H\&E) staining. At least three samples from each group were evaluated. The tissue sections were visualized under light microscope (Olympus BX60, Tokyo, Japan).

\section{Estimation of cardiac damage}

In present study, heart tissue injury was assessed by determining the concentration of LDH and $\mathrm{CK}-\mathrm{MB}$ in the perfusate. The $\mathrm{LDH}$ and $\mathrm{CK}-\mathrm{MB}$ content in the perfusate were measured using the assay kit following the manufacturer's instructions. Samples of the perfusate were collected from the isolated heart at 25, 63, 90 and 120 min of perfusion.

\section{Measurements of anti-oxidant indices}

The hearts tissue was cut into small pieces of tissue and then was ground with lysate buffer by using a glass homogenizer. Supernatant of tissue homogenate was frozen for each tissue analysis. MDA, SOD and GSH-PX activity were assessed using commercial ELISA kits following the manufacturer's instructions. All enzyme activities were normalized to the total protein concentrations, which were determined using a bicinchoninic acid (BCA) protein assay kit (Beyotime, Shanghai, China).

\section{Estimation of Cyt-c, Bcl-2 and Bax levels}


184

185

186

187

188

189

190

191

192

193

194

195

196

197

198

199

200

201

202

203

204

205

206

207

208

209

210

211

212

213

214

215

216

217

218

219

\section{0}

221

222

Following the instructions of nuclear and cytoplasmic protein extraction kit (Beyotime, Shanghai, China), heart tissue samples were weighed, minced into small pieces and homogenized using a glass homogenizer on ice $(\mathrm{w}: \mathrm{v}=1: 30,1 \mathrm{~mL}$ lysis buffer was added in 30 $\mathrm{mg}$ tissue sample). The homogenates were centrifuged at $1500 \times \mathrm{g}$ for $5 \mathrm{~min}$ at $4{ }^{\circ} \mathrm{C}$ and the cytoplasmic protein was obtained from the supernatant. The Cyt-c, Bcl-2 and Bax protein levels were measured according to the manufacturer's instructions of commercial kits through an ELISA-type method (Cloud Clone Corp, Houston, USA).

\section{Western blotting analysis}

Myocardial tissue samples were lysed with RIPA buffer containing protease inhibitors for 15 min on ice. The total lysates were clarified by centrifugation, and supernatants were collected. Protein samples (20-25 mg per lane) were loaded on the gels and then separated by $10 \%$ sodium dodecyl sulfate-polyacrylamide gel electrophoresis (SDS-PAGE) under reducing conditions and transferred onto the nitrocellulose membrane (Roche, Mannheim, Germany). The membrane was washed with PBS with $0.1 \%$ Tween-20 (PBST) and blocked with 5\% skim milk in shaking table for $2 \mathrm{~h}$ at room temperature. Then the membrane was washed with PBST and incubated with antigen-specific rabbit IgG antibodies (anti-caspase 3 and 9, anti- $\beta$-actin; Abcam) diluted to 1:1000 in PBST. Next, the membrane was washed with PBST and incubated with fluorescentlabeled goat anti-rabbit secondary antibodies (LI-COR, Lincoln, Nebraska, USA) diluted to 1:2500 in PBST for $2 \mathrm{~h}$ at $4{ }^{\circ} \mathrm{C}$. The target protein bands were scanned using the blot imaging system GelLogic 212 PRO (Carestream, Rochester, NY, USA) after washing with PBST. The obtained images were quantified as the final results by image J 1.4.3 (www.imagej.nih.gov/ij). The results were expressed as the fold induction, which were than compared with the normal control.

\section{TUNEL assay}

Heart sections of $3 \mu \mathrm{m}$ thickness were obtained using a microtome. The sections were deparaffinized in xylene and rehydrated in concentration gradient of ethanol $(100 \%, 95 \%, 75 \%)$. Following this, sections were then incubated with proteinase $\mathrm{K}$ and with $30 \% \mathrm{H}_{2} \mathrm{O}_{2}$ to enhance tissue permeability and diminish any endogenous peroxidase activity respectively. Apoptosis was determined using a terminal deoxynucleotidyl transferase-mediated dUTP-X nick end labeling (TUNEL) assay kit (Roche, Basel, Switzerland) according to the manufacturer's protocol. The sections were incubated with complete labeling reaction buffer for $60 \mathrm{~min}$ and antibody solution for $30 \mathrm{~min}$. Chromogenic reaction was visualized using 3,3-diaminobenzidine (DAB). Sections were visualized under light microscope (Olympus BX60, Tokyo, Japan).

\section{Statistical Analysis}

SPSS16.0 for Windows (SPSS Inc., Chicago, USA) was used for statistical analysis. All data were expressed as mean \pm standard deviation (SD). For comparisons between groups, the one- 
223

224

225

226

227

228

229

230

231

232

233

234

235

236

237

238

239

240

241

242

243

244

245

246

247

248

249

250

251

252

253

254

255 way ANOVA or student T-test was used where appropriate. Statistical differences were considered significant at $\mathrm{P}<0.05$. \# $\mathrm{P}<0.05$ and \#\# $\mathrm{P}<0.01$ vs. control group. ${ }^{*} \mathrm{P}<0.01$ and $* * \mathrm{P}<0.001$ vs. $\mathrm{I} / \mathrm{R}$ group.

\section{RESULTS}

\section{Effects of TAX on Cardiac Parameters of Isolated Hearts}

We examined whether TAX could protect the hearts of rat against ex vivo I/R injury. Results showed no obvious alteration in the average HR of isolated heart during reperfusion with or without TAX. In addition, no significant HR change was observed between I/R and normal groups during the $30 \mathrm{~min}$ of ischemia and $60 \mathrm{~min}$ of reperfusion (Fig. 2A). After reperfusion, $\mathrm{LVDP},+\mathrm{dp} / \mathrm{dt}_{\max }$ and $-\mathrm{dp} / \mathrm{dt}_{\min }$ from different treatment groups decreased in varying degrees. For instance, LVDP was significantly increased in TAX $15 \mu \mathrm{M}$ group compared with $\mathrm{I} / \mathrm{R}$ group at the end periods of reperfusion ( $\mathrm{LVDP}=68 \mathrm{mmHg}$ vs. $52 \mathrm{mmHg}, \mathrm{P}<0.05$, Fig. 2B). In comparison with $\mathrm{I} / \mathrm{R}$ group, treatment with $15 \mu \mathrm{M}$ TAX significantly improved the RPP in rat at $60 \mathrm{~min}$ of reperfusion (RPP $=15294 \mathrm{mmHg} \times$ beats $/ \mathrm{min}$ in TAX vs. $10643 \mathrm{mmHg} \times$ beats $/ \mathrm{min}$ in $\mathrm{I} / \mathrm{R}, \mathrm{P}<0.01$, Fig. $2 \mathrm{E}$ ). Results showed that TAX treatment improved the cardiac function recovery of rats during myocardial $\mathrm{I} / \mathrm{R}$ injury.

\section{TAX Down-regulated the Release of LDH and CK-MB}

At different time points of perfusion, heart effluents were collected. The LDH level in the whole perfusion process was not conspicuously altered in control group. The perfusate LDH activity of $\mathrm{I} / \mathrm{R}$ group was improved compared with that of control group after reperfusion and was significantly increased at $60 \mathrm{~min}$ of reperfusion $(\mathrm{P}<0.01)$. TAX highly reduced the LDH levels compared with $\mathrm{I} / \mathrm{R}$ group at $60 \mathrm{~min}$ of reperfusion (Fig. 3A). CK-MB release was similar to the LDH release. The expression in the whole perfusate process was not conspicuously changed in the control group. In the perfusion stabilization, no significant difference was observed in the CK-MB level among the four groups. However, the levels in I/R group were markedly higher after 30 min of reperfusion compared with perfusion stabilization $(\mathrm{P}<0.05)$. Interestingly, the CK-MB release in both TAX 5 and $15 \mu \mathrm{M}$ group was significantly decreased at end of reperfusion compared with $\mathrm{I} / \mathrm{R}$ group $(\mathrm{P}<0.05$ or $\mathrm{P}<0.01$ ) (Fig. 3B). These results indicated that TAX could protect the cardiac function against I/R injury.

\section{Effect of TAX on Myocardial Morphology}


274

\section{5}

276

277

278

279

280

281

282

283

284

285

286

287

288

289

Histopathological examination of myocardial tissue was assessed by H\&E staining. Typical micrographs of the myocardial structure are shown in Fig. 4. In control group (Fig. 4A), The morphology of the myocardial tissue was normal. Cardiomyocytes are arranged closely, the intercellular space is small, and edema does not exist between cells. By contrast, I/R group (Fig. 4B) showed degenerated muscle fibers and obvious contraction band, severe obvious cells edema, many infiltrated inflammatory cells. Fig. $4 \mathrm{C}$ shows that the TAX $5 \mu \mathrm{M}$ group maintained the myocardium with only slightly irregularly arranged fibers and a few contraction bands. Fig. 4D shows that the TAX $15 \mu \mathrm{M}$ group showed orderly cardiomyocytes but a few cell dissolution and degeneration. Results showed that treatment with $15 \mu \mathrm{M}$ TAX significantly reduced $\mathrm{I} / \mathrm{R}$ injury compared with I/R group.

\section{Effect of TAX on I/R-induced Oxidative Stress in the Myocardium}

To explore the cardio-protective mechanism of TAX, the effects of TAX on SOD, GSH-PX and MDA activity were investigated in myocardial tissue in response to I/R injury. Fig. 5 shows that TAX $15 \mu \mathrm{M}$ group, the SOD and GSH-PX activity were increased significantly compared with those of in $\mathrm{I} / \mathrm{R}$ group $(\mathrm{P}<0.01)$, whereas no significant difference was observed in the TAX $5 \mu \mathrm{M}$ group. Conversely, these TAX treatment groups showed that MDA production was significantly reduced $(\mathrm{P}<0.01)$ compared with $\mathrm{I} / \mathrm{R}$ group.

\section{TAX Protects Myocardial Cell from I/R-induced Mitochondrial Damage}

To evaluate if the effect of TAX is mediated through attenuation of the mitochondrial damage, we determined cytochrome c (Cyt-c) in cytosol. Fig. 6A shows that $\mathrm{I} / \mathrm{R}$ increased the cytosolic Cyt-c levels $(\mathrm{P}<0.01)$. By comparison, TAX at different doses could reduce $\mathrm{I} / \mathrm{R}$-induced increase of Cyt-c levels $(\mathrm{P}<0.01)$. The result suggested that TAX attenuated $\mathrm{I} / \mathrm{R}$ induced Cyt-c release to the cytosol. The Bcl-2 family of proteins modulates the release of Cyt-c by regulating mitochondrial transmembrane potential. We also determined Bcl-2 and Bax protein levels. Compared with the control group, I/R down-regulated Bcl-2 but up-regulated Bax protein expression (Figs. 6B-6C). TAX increased Bcl-2 levels but decreased Bax levels compared with $\mathrm{I} / \mathrm{R}$. These results indicate that TAX may protect mitochondria against apoptosis by regulating the expression of the Bcl-2 family proteins.

\section{TAX Attenuates Myocardial I/R-induced Apoptosis}

To explore the potential mechanism of TAX in rats subjected to I/R-induced myocardial injury, we investigated the protein expressions of active-caspase 3 and 9. Compared with control 
290

291

292

293

294

295

296

297

298

299

300

301

302

303

304

305

306

307

308

309

310

311

312

313

314

315

316

317

318

319

320

321

322

323

324

325

326

327

group, the expression of active-caspase 3 was up-regulated in I/R group. While compared with I/R group, TAX treatment group significantly reduced the level of active-caspase 3 (Fig. 7C). In TAX treatment group, the expression of active-caspase 9 was down-regulated in $15 \mu \mathrm{M}(\mathrm{P}<$ 0.05), but did not change significantly in $5 \mu \mathrm{M}$ (Fig. 7D). In addition, TUNEL assay was performed to evaluate the effect TAX on myocardial apoptosis. An increased number of TUNEL positive cells were observed in I/R group in comparison to control group. Furthermore, a less number of TUNEL positive cell was present in the TAX treatment group (Fig. 8). Results indicated that TAX inhibited apoptotic level in heart injury induced by I/R.

\section{DISCUSSION}

Growing evidence indicates a therapeutic action of TAX in cardiovascular disease. However, the implications of TAX in I/R injury remain unclear. To the best of our knowledge, this study was the first to evaluate the cardioprotective effects of TAX in isolated rat heart subjected to I/R injury. We demonstrated that an important role of TAX in improving cardiac function, and inhibiting oxidative stress and apoptosis in a model of I/R injury in vitro.

In the late 19th century, Oscar Langendorff pioneered the isolated perfused heart model. Since then, the procedure has been used to probe the pathophysiology of $I / R$ with the dawn of molecular biology (Bell et al., 2011). Today, the Langendorff heart assay is a predominant technique in vitro, which is used in physiological and pharmacological research. It allows the examination of cardiac contractile strength and heart rate without complications of an intact animal (Herr et al., 2015). Therefore, we determined the effect of TAX to the cardiac function parameters of isolated heart in myocardial $\mathrm{I} / \mathrm{R}$ injury by using the Langendorff equipment. Cardiac functions mainly depend on the contraction and relaxation properties of the ventricular muscle. Changes in cardiac function are strongly linked to the severity of I/R injury (Mehdizadeh et al., 2013). The data from our analyses showed that $\mathrm{I} / \mathrm{R}$ can cause marked myocardial dysfunction, including the reduction of LVDP, $+\mathrm{dp} / \mathrm{dt}_{\max }$ and $-\mathrm{dp} / \mathrm{dt}_{\min }$. TAX treatment significantly improved cardiac diastolic dysfunction but did not alter the average HR in isolated heart.

LDH is a marker of cellular damage and common disease due to its mass release to plasma during tissue injuries, such as heart failure. CK-MB, which is expressed extensively in cardiac myocyte, was often tested in the serum as an indicator of rhabdomyolysis damage, myocardial damage and acute kidney injury in clinic (Moghadam-Kia et al., 2016). The increase in LDH and CK-MB levels in the organ perfusate after ischemia is a direct evidence of cardiac damage (Houshmand et al., 2009). In comparison with control group, LDH and CK-MB activity were significantly increased in I/R group during myocardial I/R injury. Perfusate LDH and CK-MB activity in the TAX treatment groups, particularly in TAX $15 \mu \mathrm{M}$ group, were remarkably reduced compared with those in I/R group, which is consistent with the observation of changing cardiac function parameters. In addition, histopathological examination was implemented to 
328

329

330

331

332

333

334

335

336

337

338

339

340

341

342

343

344

345

346

347

348

349

350

351

352

353

354

355

356

357

358

359

360

361

362

363

364

365

366

assess the effect of TAX on myocardial morphology. The results of pathomorphological research in the heart samples in $\mathrm{I} / \mathrm{R}$ group show acute myocardial damage, and TAX causes favorable morphological changes in the heart during $I / R$ injury. These results supported the potential application of TAX as a cardioprotective agent in myocardial I/R injury.

Under normal conditions, tissues could maintain the balance between generation and clearance of ROS. However, the balance is disrupted during I/R and causes significant increase in ROS (Becker et al., 2004). Excess ROS can oxidize lipids, proteins and DNA, which cause dysfunction of these molecules, resulting in the degeneration of tissue function (Kleikers et al., 2012). Minimizing the ROS production is an important strategy to prevent cardiomyocyte $I / R$ injury (He et al., 2016). Therefore, the activation of the anti-oxidant enzyme system is necessary to reduce oxidative stress-induced tissue damage (Matsushima et al, 2013). The SOD and GSHPX rate are used to evaluate tissue per-oxidative injury (Maciejczyk et al., 2017). In addition, MDA is an index to evaluate the severity of lipid peroxidation, which is produced by lipid peroxidation, resulting in the destruction of structural proteins and cellular structures (Pizzimenti et al., 2013). Our results showed that SOD and GSH-PX activities were conspicuously increased, whereas MDA level was dramatically decreased by TAX, especially in TAX $15 \mu \mathrm{M}$ group. Therefore, TAX exhibited the cardioprotective effects by enhancing the antioxidase activity and inhibiting free radical peroxidation.

Mitochondrial damage plays an important role in I/R-induced injury. It is the final arbitrator for I/R-induced cell apoptosis (Powers et al., 2007). During ischemia and mainly during the early period of reperfusion, excessive ROS causes myocardial $\mathrm{Ca}^{2+}$ overload and the opening of the mitochondrial permeability transition pore (mPTP), which can reduce mitochondrial function and finally result in an increase in myocardial cell apoptosis (Garciarena et al., 2011; Halestrap et al., 2015). One of the ways of cell apoptosis is activated by the release of Cyt-c from the mitochondria to the cytosol. In our study, results showed that TAX can weaken the observed increase in the expression of Cyt-c in cytosol. It is very likely that the increased cytosolic content of Cyt-c, which mediates apoptosis, while its expression in mitochondria was not changed (Lundberg et al., 2004). Further investigation of the pathological changes in myocardial tissues by TUNEL assays showed different degrees of apoptosis. Results indicated a positive effect of TAX in the inhibition of apoptosis. Therefore, it can make an assumption that down-regulation of Cyt-c result from TAX attenuated apoptotic processes.

As an important mitochondrial regulator during myocardial apoptosis, Bcl-2 exerts antiapoptotic effects by blocking the release of Cyt-c and reducing caspase activity. Apoptosisrelated proteins, caspase 3 and 9, also play crucial roles in apoptosis. The caspase apoptotic pathway responds to death signals by releasing apoptosis-inducing factor from the mitochondria, which were then translocated to the nucleus (D'Amelio et al., 2012). In this study, Bcl-2, an antiapoptotic protein and Bax, a pro-apoptotic protein were used to assess the effects of TAX on cardiomyocytes apoptosis. The result demonstrated that TAX treatment increased the protein expression of $\mathrm{Bcl}-2$, and significantly reduced the Bax expression compared with $\mathrm{I} / \mathrm{R}$ group. 
367

368

369

370

371

372

373

374

375

376

377

378

379

380

381

382

383

384

385

386

387

388

389

390

391

392

393

394

395

396

397

398

399

400

401

402

Caspase 3 and 9 were tested to measure the apoptotic level in the isolated heart after I/R injury. We found that the increased expression of the active form of caspase 3 and 9 under ischemic conditions and their expression were decreased in TAX group. Consistent with these results, treatment with TAX significantly decreased myocardial apoptosis by regulating the expression of apoptosis-related proteins, including Bax, Bcl-2 and caspase 3 and 9. These findings suggest that the inhibition of apoptosis is closely related to the underlying beneficial effect of TAX in I/R injury. Cardiomyocytes death occurs during $\mathrm{I} / \mathrm{R}$ injury by apoptosis, by necrosis and in association with autophagy (Whelan et al., 2010; Gatica D et al., 2015). In this study, we studied the effect of apoptosis on myocardial injury, but the effect of other pathways on myocardial injury was not excluded, which requires further exploration.

\section{CONCLUSIONS}

In conclusion, TAX exerted cardioprotective effects against I/R injury by inhibiting oxidative stress and cardiac myocyte apoptosis. The underlying mechanism for these phenomena may involve modulation of mitochondrial apoptosis pathway. Our finding provides a novel thought for therapeutic development as an adjuvant therapy to I/R injury.

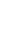

\section{REFERENCES}

Akhlaghi M, Bandy B. 2009. Mechanisms of flavonoid protection against myocardial ischemiareperfusion injury. Journal Molecular Cellular Cardiology 46(3):309-317.

Arutyunyan TV, Korystova AF, Kublik LN, Levitman MKh, Shaposhnikova VV, Korystov YN. 2016. Taxifolin and fucoidin abolish the irradiation-induced increase in the production of reactive oxygen species in rat aorta. Bulletin of Experimental Biology and Medicine 160(5):635638.

Barteková M, Carnická S, Pancza D, Ondrejcáková M, Breier A, Ravingerová T. 2010. Acute treatment with polyphenol quercetin improves postischemic recovery of isolated perfused rat heart after global ischemia. Canadian Journal of Physiology and Pharmacology 88(4):465471.

Becker LB. 2004. New concepts in reactive oxygen species and cardiovascular reperfusion physiology. Cardiovascular Research 61(3):461-470.

Bell RM, Mocanu MM, Yellon DM. 2011. Retrograde heart perfusion: the Langendorff technique of isolated heart perfusion. Journal of Molecular and Cellular Cardiology 50(6):940950.

Bjorklund G, Dadar M, Chirumbolo S, Lysiuk R. 2017. Flavonoids as detoxifying and prosurvival agents: what's new? Food and Chemical Toxicology 110:240-250.

Braunwald E. 2012. The treatment of acute myocardial infarction: the past, the present, and the 
403 future. European Heart Journal of Acute Cardiovascular Care 1(1):9-12.

404 Carden DL, Granger DN. 2000. Pathophysiology of ischaemia-reperfusion injury. Journal of 405 Pathology 190: 255-266.

406 Chong SJ, Low IC, Pervaiz S. 2014. Mitochondrial ROS and involvement of Bcl-2 as a 407 mitochondrial ROS regulator. Mitochondrion 19:39-48.

408 D'Amelio M, Sheng M, Cecconi F. 2012. Caspase-3 in the central nervous system: beyond 409 apoptosis. Trends in Neurosciences 35(11):700-709.

410 Dianzani C, Lepore A, Gentile F, Barrera G. 2013. Interaction of aldehydes derived from lipid 411 peroxidation and membrane proteins. Frontiers in Physiology 4:242.

412 Du Y, Plante E, Janicki JS, Brower GL. 2010. Temporal evaluation of cardiac myocyte 413 hypertrophy and hyperplasia in male rats secondary to chronic volume overload. American 414 Journal of Pathology 177(3): 1155-1163.

415 Garciarena CD, Fantinelli JC, Caldiz CI, Chiappe de Cingolani G, Ennis IL, Perez NG, 416 Cingolani HE, Mosca SM. 2011. Myocardial reperfusion injury: reactive oxygen species vs. 417 NHE-1 reactivation. Cellular Physiology and Biochemistry 27(1):13-22.

418 Gatica D, Chiong M, Lavandero S, Klionsky DJ. 2015. Molecular mechanisms of autophagy 419 in the cardiovascular system. Circulation Research 116:456-467.

420 Giordano FJ. 2005. Oxygen, oxidative stress, hypoxia, and heart failure. Journal Clinical 421 Investigation 115(3):500-508.

422 Gottlieb RA, Burleson KO, Kloner RA, Babior BM, Engler RL. 1994. Reperfusion injury 423 induces apoptosis in rabbit cardiomyocytes. Journal of Clinical Investigation 94(4): 1621-1628.

424 Guo H, Zhang X, Cui Y, Zhou H, Xu D, Shan T, Zhang F, Guo Y, Chen Y, Wu D. 2015. 425 Taxifolin protects against cardiac hypertrophy and fibrosis during biomechanical stress of 426 pressure overload. Toxicology and Applied Pharmacology 287(2):168-177.

427 Halestrap AP, Richardson AP. 2015. The mitochondrial permeability transition: a current 428 perspective on its identity and role in ischaemia/reperfusion injury. Journal of Molecular and 429 Cellular Cardiology 78:129-141.

430 He F, Li J, Liu Z, Chuang CC, Yang W, Zuo L. 2016. Redox mechanism of reactive oxygen 431 species in exercise. Frontiers in Physiology 7:486.

432 Herr DJ, Aune SE, Menick DR. 2015. Induction and Assessment of Ischemia-reperfusion 433 Injury in Langendorff-perfused Rat Hearts. Journal of Visualized Experiments 101:e52908.

434 Houshmand F, Faghihi M, Zahediasl, S. 2009. Biphasic protective effect of oxytocin on 435 cardiac ischemia/reperfusion injury in anaesthetized rats. Peptides 30(12):2301-2308.

436 Kleikers PW, Wingler K, Hermans JJ, Diebold I, Altenhöfer S, Radermacher KA, Janssen 437 B, Görlach A, Schmidt HH. 2012. NADPH oxidases as a source of oxidative stress and 438 molecular target in ischemia/reperfusion injury. Journal of Molecular Medicine (Berlin) 439 90(12):1391-1406.

440 Konstantinidis K, Whelan RS, Kitsis RN. 2012. Mechanisms of cell death in heart disease. 441 Arteriosclerosis Thrombosis Vascular Biology 32(7):1552-1562. 
442 Lundberg KC, Szweda LI. 2004. Initiation of mitochondrialmediated apoptosis during cardiac 443 reperfusion. Archives of Biochemistry and Biophysics 432(1):50-57.

444 Maciejczyk M, Mikoluc B, Pietrucha B, Heropolitanska-Pliszka E, Pac M, Motkowski R, 445 Car H. 2017. Oxidative stress, mitochondrial abnormalities and antioxidant defense in Ataxia446 telangiectasia, Bloom syndrome and Nijmegen breakage syndrome. Redox Biology 11:375-383.

447 Matsushima S, Kuroda J, Ago T, Zhai P, Ikeda Y, Oka S, Fong GH, Tian R, Sadoshima J. 448 2013. Broad suppression of NADPH oxidase activity exacerbates ischemia/reperfusion injury 449 through inadvertent downregulation of HIF-1 $\alpha$ and upregulation of PPAR- $\alpha$. Circulation 450 Research 112(8):1135-1149.

451 Mehdizadeh R, Parizadeh MR, Khooei, AR, Mehri S, Hosseinzadeh H. 2013. 452 Cardioprotective effect of saffron extract and safranal in isoproterenol-induced myocardial 453 infarction in wistar rats. Iranian Journal of Basic Medical Sciences 16(1):56-63.

454 Moghadam-Kia S, Oddis CV, Aggarwal R. 2016. Approach to asymptomatic creatine kinase 455 elevation. Cleveland Clinic Journal of Medicine 83(1):37-42.

456 Moon YJ, Wang X, Morris ME. 2006. Dietary flavonoids: Effects on xenobiotic and 457 carcinogen metabolism. Toxicology in Vitro 20(2):187-210.

458 Naito H, Furukawa Y, Chino D, Yamada C, Hashimoto K. 2000. Effects of zatebradine and 459 propranolol on canine ischemia and reperfusion-induced arrhythmias. European Journal of 460 Pharmacology 388(2):171-176.

461 Pangonyte D, Stalioraityte E, Ziuraitiene R, Kazlauskaite D, Palubinskiene J, Balnyte I. 462 2008. Cardiomyocyte remodeling in ischemic heart disease. Medicina (Kaunas) 44(11):848-854. 463 Pizzimenti S, Ciamporcero E, Daga M, Pettazzoni P, Arcaro A, Cetrangolo G, Minelli R, 464 Powers SK, Murlasits Z, Wu M, Kavazis AN. 2007. Ischemia-reperfusion-induced 465 cardiacinjury: a brief review. Medicine and Science in Sports and Exercise 39(9):1529-1536.

466 Raj Narayana K, Sripal Reddy M, Chaluvadi MR, Krishna DR. 2001. Bioflavonoids 467 classification, pharmacological, biochemical effects and therapeutic potential. Indian Journal of 468 Pharmacology 33:2-16

469 Shanmugam K, Ravindran S, Kurian GA, Rajesh M. 2018. Fisetin confers cardioprotection 470 against myocardial ischemia reperfusion injury by suppressing mitochondrial oxidative stress 471 and mitochondrial dysfunction and inhibiting glycogen synthase kinase $3 \beta$ activity. Oxidative 472 Medicine and Cellular Longevity

473 Sun X, Chen RC, Yang ZH, Sun GB, Wang M, Ma XJ, Yang LJ, Sun XB. 2014. Taxifolin 474 prevents diabetic cardiomyopathy in vivo and in vitro by inhibition of oxidative stress and cell 475 apoptosis. Food and Chemical Toxicology 63:221-232.

476 Wang YY, Yuan Y, Wang XC, Wang YF, Cheng J, Tian L, Guo XH, Qin DM, Cao WJ. 477 2017. Tilianin post-conditioning attenuates myocardial ischemia/reperfusion injury via 478 mitochondrial protection and inhibition of apoptosis. Medical Science Monitor 18(23):4490-4499.

479 Whelan RS, Kaplinskiy V, Kitsis RN. 2010. Cell death in the pathogenesis of heart disease: 480 mechanisms and significance. Annual Review of Physiology 72:19-44. 
481 Xie X, Feng J, Kang Z, Zhang S, Zhang L, Zhang Y, Li X, Tang Y. 2017. Taxifolin protects 482 RPE cells against oxidative stress-induced apoptosis. Molecular Vision 23:520-528.

483 Xuan FF, Jian J. 2016. Epigallocatechin gallate exerts protective effects against myocardial 484 ischemia/reperfusion injury through the PI3K/Akt pathway-mediated inhibition of apoptosis and 485 the restoration of the autophagic flux. International Journal of Molecular Medicine 38(1):328486336.

487 Zhao H, Sapolsky RM, Steinberg G. 2006. Interrupting reperfusion as a stroke therapy: 488 ischemic postconditioning reduces infarct size after focal ischemia in rats. Journal of Cerebral 489 Blood Flow and Metabolism 26(9):1114-1121. 


\section{Figure 1}

Figure 1 Experimental protocol

Figure 1. Schematic diagram of experimental protocol. Normal control group (Control); Myocardial I/R control group (I/R); I/R + TAX treatment group (TAX $5 \mu \mathrm{M}) ; \mathrm{I} / \mathrm{R}+\mathrm{TAX}$ treatment group (TAX $15 \mu \mathrm{M})$.

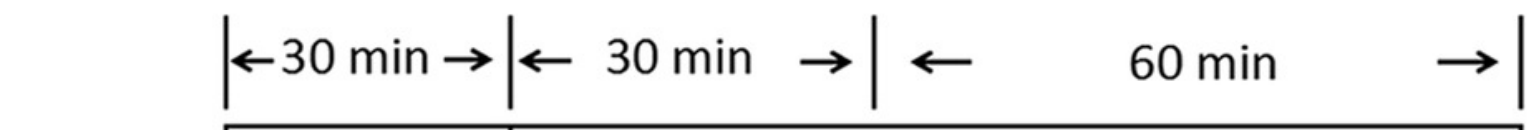

Control

\begin{tabular}{|l|l|}
\hline Baseline & Reperfusion \\
\hline
\end{tabular}

I/R

\begin{tabular}{|l|l|l|}
\hline Baseline & Ischemia & Reperfusion \\
\hline
\end{tabular}

$\mathrm{I} / \mathrm{R}+\mathrm{TAX} 5 \mu \mathrm{M}$

\begin{tabular}{|l|l|ll|}
\hline Baseline & Ischemia & $\boldsymbol{\Lambda}$ & Reperfusion \\
\hline
\end{tabular}

I/R + TAX $15 \mu \mathrm{M}$

\begin{tabular}{|l|l|ll|}
\hline Baseline & Ischemia & N & Reperfusion \\
\hline
\end{tabular}

A Taxflion and $\mathrm{K}-\mathrm{H}$ solution for $60 \mathrm{~min}$ after $30 \mathrm{~min}$ of whole heart ischemia 
Figure 2

Figure 2. TAX treatment improved the cardiac function recovery of rats during myocardial I/R injury in vitro model.

(A) Heart rate (HR, beat/per min); (B) left ventricular developed pressure (LVDP, $\mathrm{mmHg}$ ); (C) maximum rate of left ventricular pressure $\left(+d p / d t_{\max }, \mathrm{mmHg} / \mathrm{s}\right)$; (D) minimum rate of increase of left ventricular pressure (-dp/dt $\left.\mathrm{din}_{\text {n, }} \mathrm{mmHg} / \mathrm{s}\right)$; (E) Rate pressure product (RPP, $\mathrm{mmHg} \times$ bpm); (F) representative left ventricular pressure records in experimental protocol form different experiment groups. 

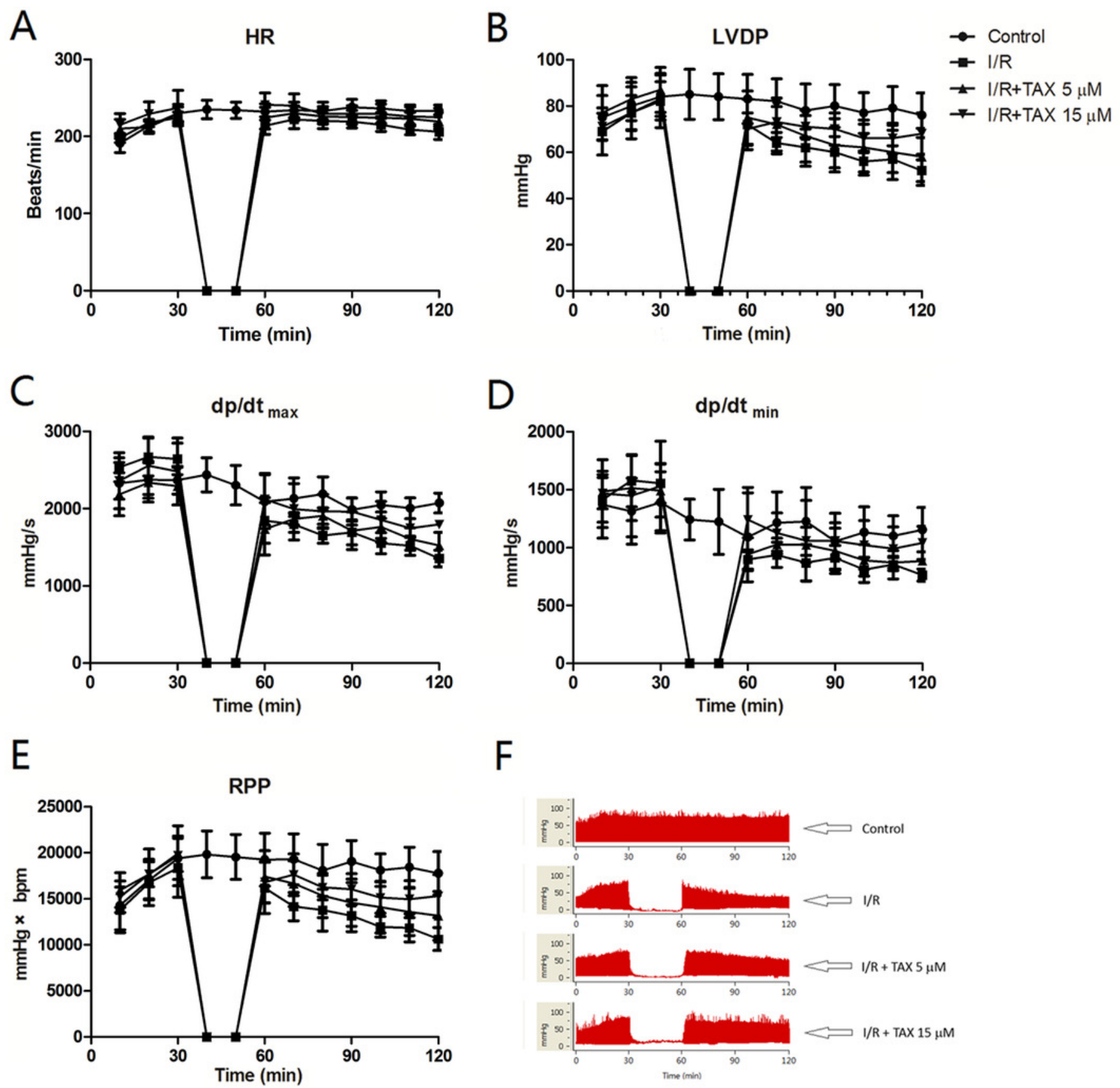
Figure 3

Figure 3. Effect of TAX on injury of cardiomyocytes by measurement of LDH and CK-MB.

The levels of perfusate LDH and CK at different time points in the control, I/R and TAX-treat group ( $5 \mu \mathrm{M}$ and $15 \mu \mathrm{M}$ ) are shown. \# $\mathrm{P}<0.05$ and \#\# $\mathrm{P}<0.01$ compared with the control group; $* \mathrm{P}<0.05$ and $* * \mathrm{P}<0.01$ compared with the I/R group; U/L: international enzyme activity unit per liter.
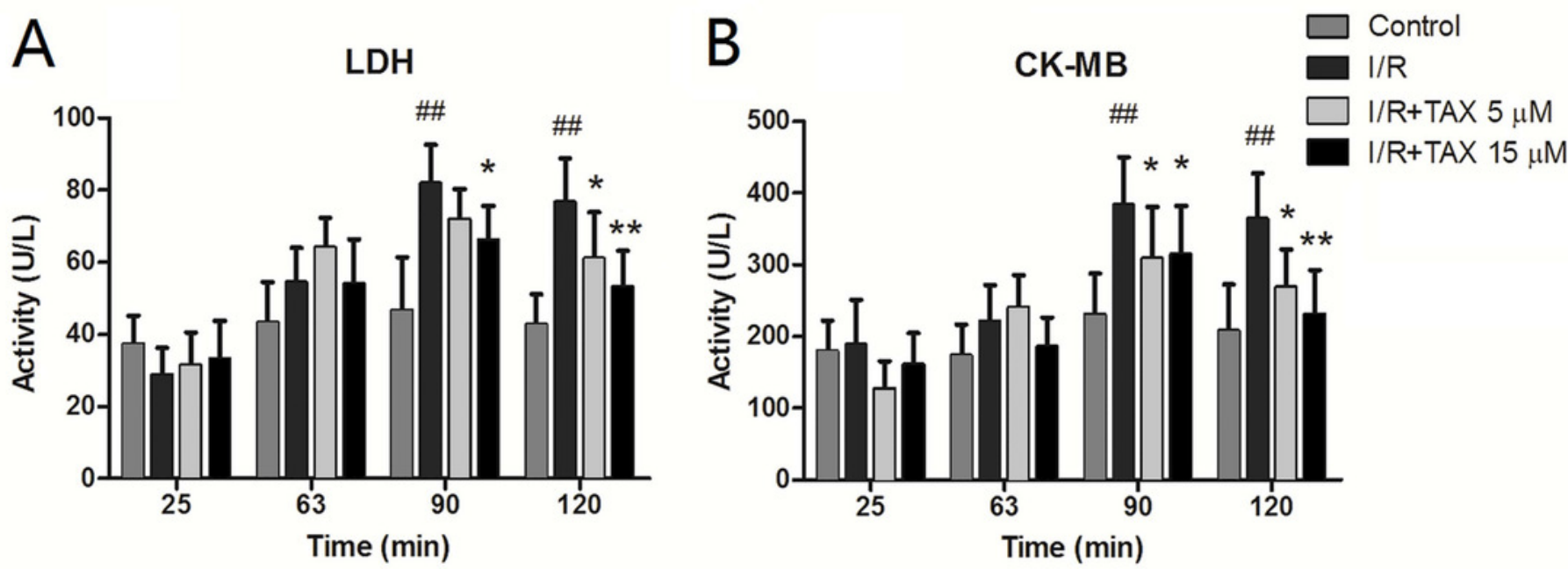


\section{Figure 4}

Figure 4. Representative micrographs of HE staining results in various experimental groups.

(A) control group; (B) I/R group; (C) TAX $5 \mu \mathrm{M}$ group; (D) TAX $15 \mu \mathrm{M}$ group, $n=3$ per group, (magnification, $\times 400),(\rightarrow)$ tissue damage and edema.
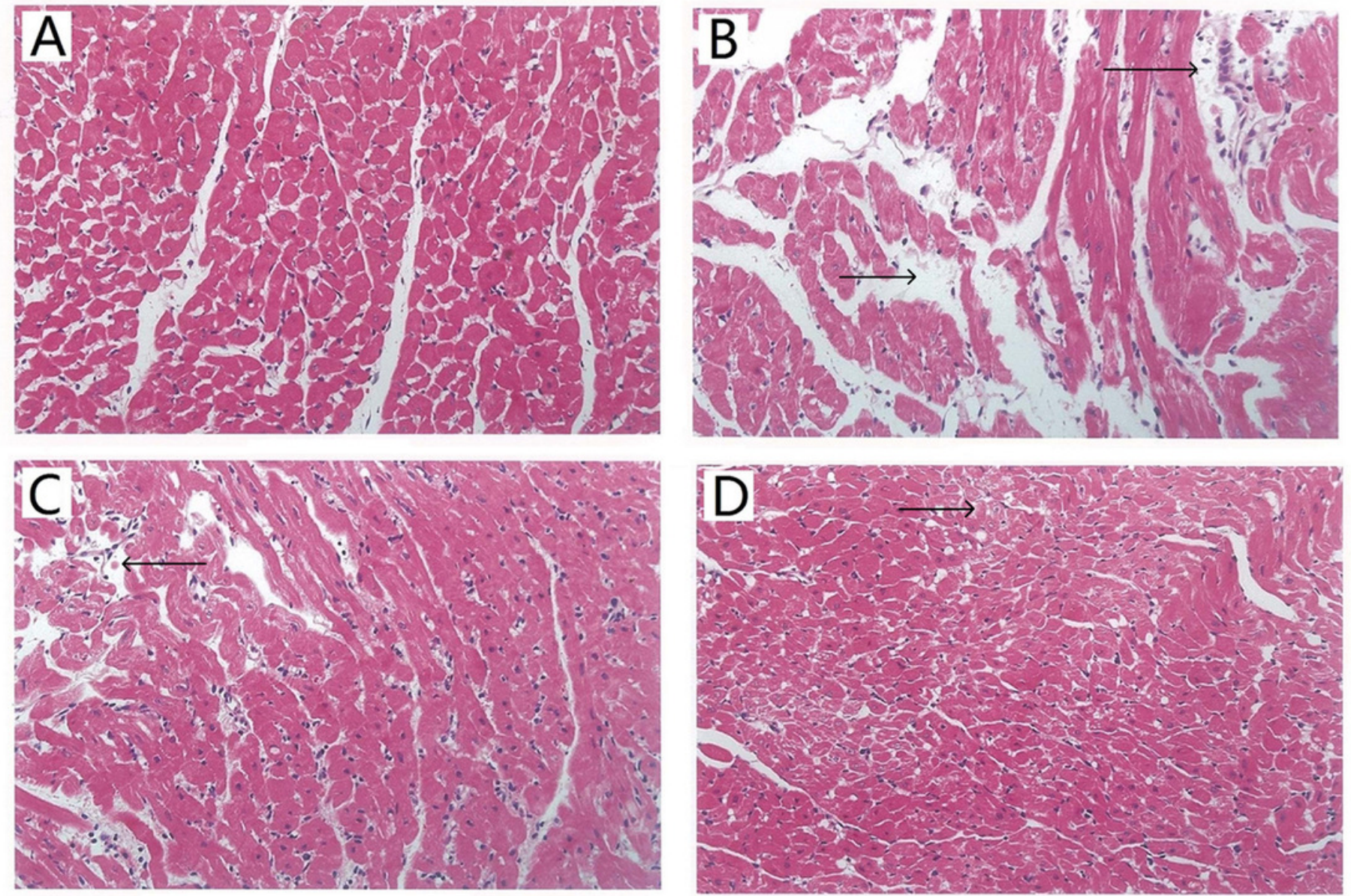


\section{Figure 5}

Figure. 5. Effect of TAX on cardiac the activity of SOD and GSH-PX, contents of MDA.

Values are presented as mean $\pm \mathrm{SD}$. \# $\mathrm{P}<0.05$ and \#\# $\mathrm{P}<0.01$ compared with the control group; $* \mathrm{P}<0.05$ and $* * \mathrm{P}<0.01$ compared with the I/R group. U/mgprot: international enzyme activity unit per milligram tissue protein.

A



B

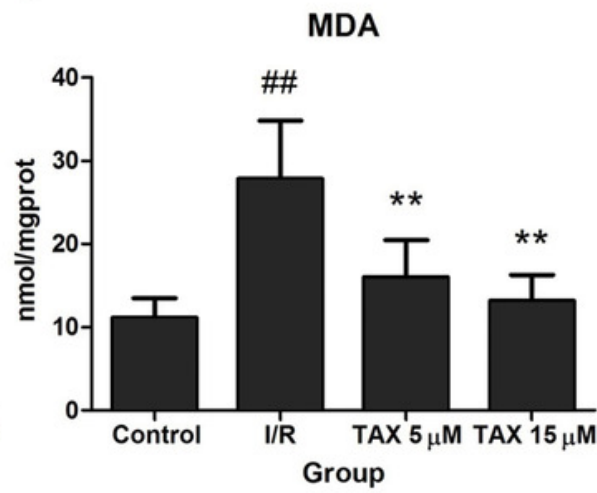

C

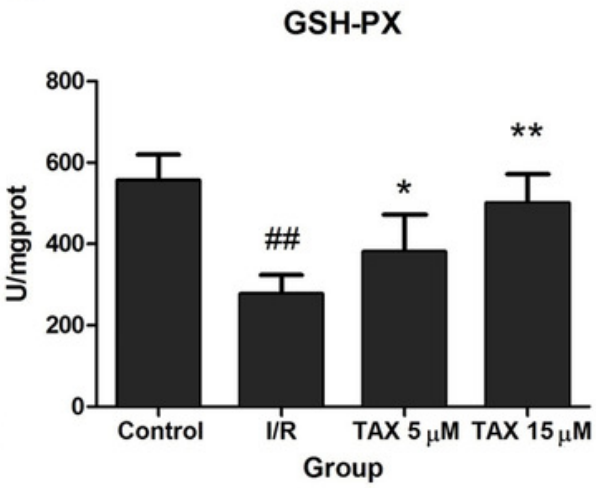


Figure 6

Figure 6. Effect of TAX on the expression of Cyt-c, Bax and Bcl-2 protein.

\# $\mathrm{P}<0.05$, \#\# $\mathrm{P}<0.01$ vs. Control group; $* \mathrm{P}<0.05$, ** $\mathrm{P}<0.01$ vs. IR group. ng/mgprot indicate the nanogram level of the target protein per milligram total protein.

A



B

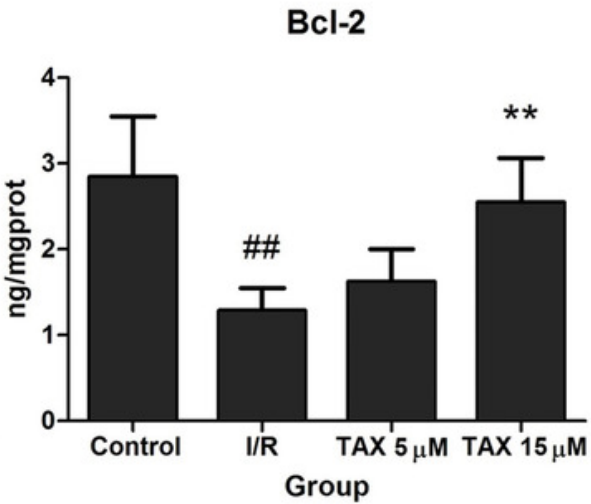

C

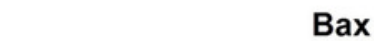




\section{Figure 7}

Fig. 7. The changes in the levels of caspase 3 , and caspase 9 at the end of reperfusion.

A

\section{Active-caspase 3}

$17 \mathrm{kDa}$

$\beta$-actin $42 \mathrm{kDa}$

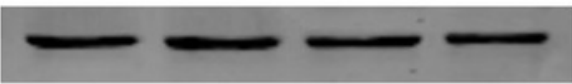

Control I/R TAX $5 \mu \mathrm{M} \operatorname{TAX} 15 \mu \mathrm{M}$

C
B

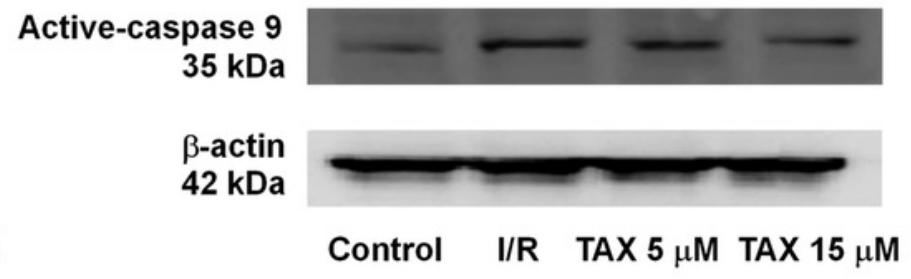

D

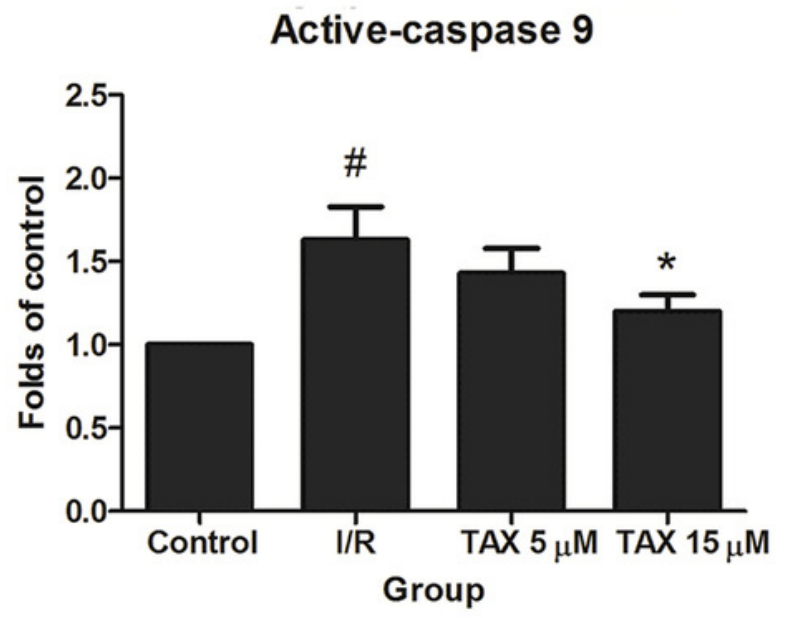


Figure 8

Figrue 8. Effect of taxifolin on cardiomyocytes apoptosis in different experimental groups.

(A) control group; (B) I/R group; (C) I/R + TAX $5 \mu \mathrm{M}$ group; (D) I/R + TAX $15 \mu \mathrm{M}$ group. $\mathrm{n}=3$ per group, 20X, Scale bar $50 \mu \mathrm{m},(\rightarrow)$ positive stain
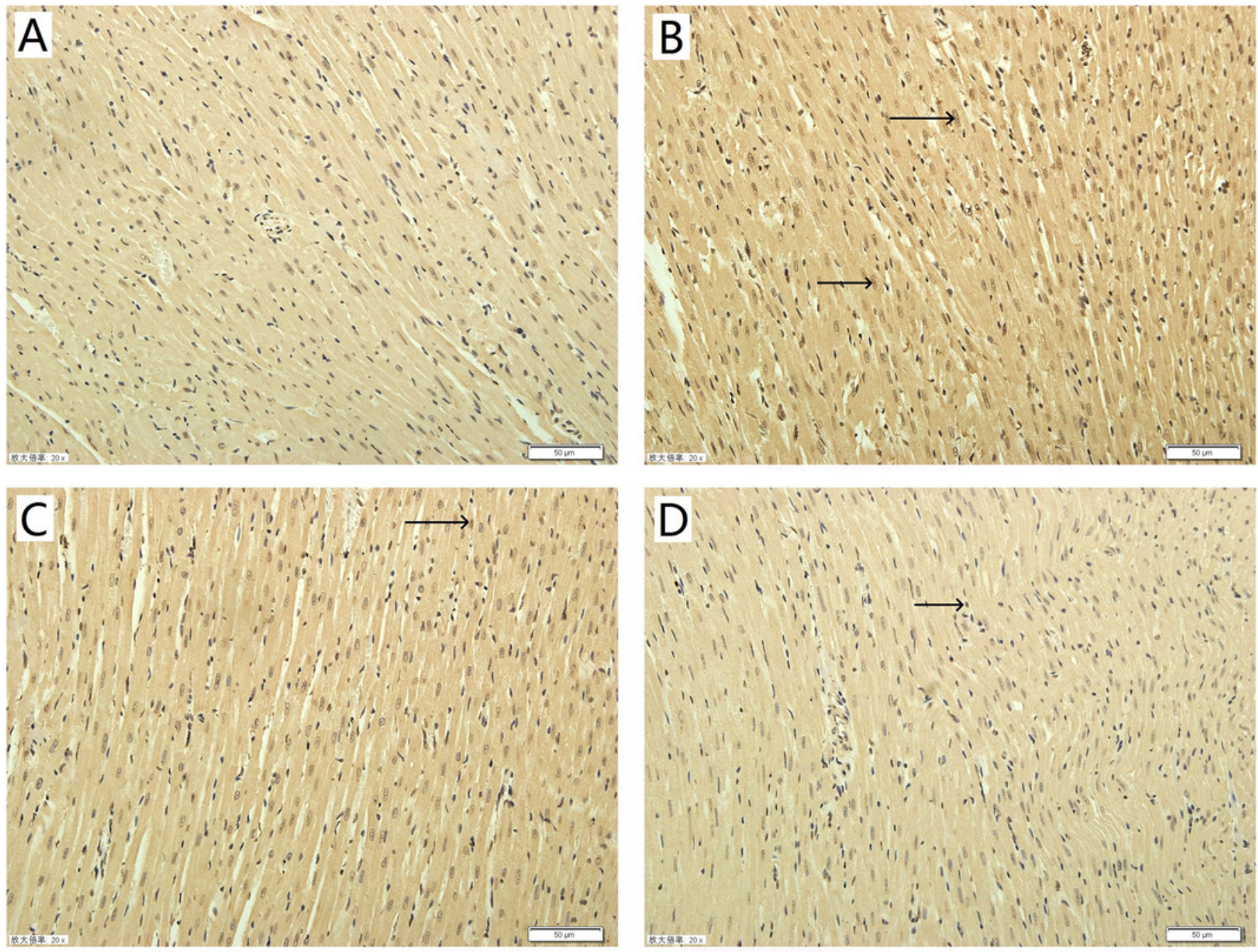